\section{Biochemistry and pharmacology of venoms}

Venoms: Chemistry and Molecular Biology. By Anthony T. Tu. Pp. 560. (Wiley-Interscience: London and New York, 1977.) $\$ 43$; $£ 25.90$.

VENOMS have always had a peculiar fascination. How do they kill animals (and, in particular, humans)? What do they contain, and can any of the components be put to use either as tools in research or as therapeutic agents? Before the advent of modern chromatographic techniques, not much progress could be made in the field but since then there has been (to say the least of it) no shortage of papers. The quality of some of these has, however, been exceedingly bad. Separation procedures still require some skill and if the skill is lacking they lead to unspecified mixtures. The desire to inject such mixtures (or, indeed, whole venoms) into animals and see what happens is (apparently) strong. It ought always to be firmly resisted.

All of this means that to write a book on venoms is a formidable undertaking. It needs a considerable knowledge of the relevant aspects of chemistry, biochemistry and pharmacology and, if one is to deal with the effects of venoms in humans, of clinical medicine as well. Enough to daunt the strongest spirit. Professor Tu admits this in his preface and uses the phrase "monumental task". He has, in the event, produced a book containing over four hundred pages devoted to snake venoms (his own research interest) and rather less than one hundred to other animal venoms (scorpions, bees, wasps, and so on). One could hold the view that this is a trifle unbalanced, since (for example) the components of bee venom have been extensively investigated both chemically and pharmacologically. On the other hand, it is true that a bewildering variety of peptides and proteins have been found in snake venoms and some of these have proved to be extremely useful. For example, the phosphodiesterase contained in many snake venoms is an invaluable tool in the determination of nucleotide sequences in nucleic acids. On thesc grounds, the long section of the book on the enzymes present in snake venoms can, no doubt, be justified.

I must confess, however, that I found the book to be essentially uncritical. Most of the papers quoted seem to be given equal weight when clearly some of them (all too many, I fear) are un- satisfactory on chemical or biological grounds or both. What, for example, is the point in giving a list (p509) of the values reported for the (so-called) $\mathbf{L D}_{50}$ of apamine in mice when it is perfectly clear that the correct value is that given by Habermann (in 1972, incidentally, and not as quoted) and subsequently confirmed by other workers? How does one feel about the (supposed) component of bee venom called "cardiopep"? Ought one not to entertain the suspicion that the biological effects ascribed to it are really due to noradrenaline (shown to be present in the venom, as distinct from the venom gland, by Banks et al., 1976, and not, as given, by Owen, 1971)? Again, what about minimine? This is certainly my favourite substance (or non-substance) because its biological activity is supposed to be the produc-

\section{Basic statistical handbook}

A Handbook of Numerical and Statistical Techniques. By J. H. Pollard. Pp.xvi+349. (Cambridge University: Cambridge, London and New York; 1977.) £13.50.

WHEN books on basic statistics continue to appear with amazing regularity, it is refreshing to find one which more than permutes the 'usual material'. This book not only describes essential statistical techniques but gives a welcome priority to elementary numerical methods.

The author aims to help computer users, firstly to understand the output of statistical packages, and secondly to adapt these and similar packages for programmable calculators to suit their own requirements. Although it is not a textbook of elementary numerical and statistical techniques, its uniformity and clarity of presentation, together with its comprehensiveness, should give it wide appeal. A basic knowledge of mathematics is assumed.

The contents have been skilfully divided into three parts. Part I (seven chapters), after a brief introduction to essential basic mathematics and a helpful discussion of sources of error, describes numerical methods for locating the roots of non-linear equations, data smoothing, integration, differentiation and interpolation. It finishes with a variety of useful techniques including the regrouping of grouped data, the fusing of smooth curves and steepest descent.

Basic statistical techniques, except regression, are presented in part II (seven chapters) which, after briefly tion of miniature flies from the larvae of Drosophilia melanogaster (whoever would think of such an experiment?) One could continue, but the list of doubtful substances and even more doubtful biological activities would be long and tedious. In defence of Professor Tu, I must say that, in my view, no one man could read and assess critically all the literature in this area.

It is a pleasure to be able to say that the book is well produced, well laid out, and that the references are remarkably up-to-date and (for the most part) remarkably complete. The book will certainly be of value (if only for the references) to workers in the field and to those wishing to enter it.

C. A. Vernon

C. A. Vernon is Professor of Chemistry at University College, London, UK.

introducing probability theory, describes the important frequency distributions including the Pearson system. Two chapters devoted to hypothesis testing and point and interval estimation provide a broad survey of parametric and distribution-free statistical tests. Each test is presented in a standard format which facilitates both comprehension and correct application. This part ends with three special topics -random numbers, data transformation, and censored and truncated distributions.

Part III (four chapters), devoted to the method of least squares, describes simple linear regression (with and without matrix notation) and most of the associated statistical tests. (Unfortunately, the statistical comparison of two or more regression lines has been omitted.) Curvilinear and multiple linear regression are presented succinctly, using matrix notation, and this part ends with an introduction to non-linear regression.

Throughout the book, each technique is separately referenced and well illustrated with a fully-worked example, usually from the life sciences. Specialised tables are sensibly interspaced with the text, while those of more general application are placed in an appendix. Every chapter includes a selection of exercises though unfortunately the answers are not given.

This handbook has been carefully assembled, it is easy to use, and crossreferencing is kept to a minimum. It should be a valuable asset to the scientist and the statistician.

A. L. Johnson

A. L. Johnson is a statistician in the $M R C$ Statistical Research and Services Unit, University College Hospital Medical School, London, UK. 